\title{
A case of two foci of primary melanoma with metastasis to the skin in giant congenital melanocytic nevi
}

\author{
Marta Fijałkowska1 ${ }^{1}$ Dariusz Nejc², Bogusław Antoszewski
}

${ }^{1}$ Department of Plastic, Reconstructive and Aesthetic Surgery, Second Chair of Surgery, Medical University of Lodz, Lodz, Poland 2Department of Surgical Oncology, Chair of Oncology, Medical University of Lodz, Lodz, Poland

Adv Dermatol Allergol 2021; XXXVIII (5): 901-902

DOI: https://doi.org/10.5114/ada.2021.110058

Congenital melanocytic nevi are moles that are present at birth or arise within the first weeks of life [1]. They vary in size, from small, medium to very large or giant ones (more than $20 \mathrm{~cm}$ in diameter) [1, 2]. Giant congenital melanocytic nevi occur in approximately 1 in 20 000-50 000 newborns [3]. It is established in the literature that patients with giant melanocytic nevus have an elevated risk of developing melanoma, which ranges from $2 \%$ to $42 \%$ in the literature [1-3]. To date, no universal guidelines to treat giant melanocytic nevi have been given [3].

The aim of this paper is to present a case of a 25-yearold female patient who developed two sites of primary melanoma and metastasis to the skin, all in the area of giant melanocytic nevi.

In March 2017, a 25-year-old female came to our OutPatient Plastic Surgery Clinic with giant congenital nevi in order to remove two distressing lesions (Figure 1). Additionally the patient is diagnosed with diabetes mellitus type 1.

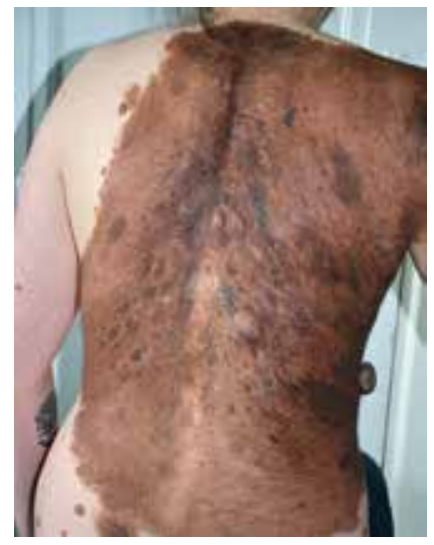

Figure 1. A 25-year-old patient with giant congenital nevi with 2 lesions on her back
Before qualification to surgery the patient was asked to go through ultrasonography procedure. The obtained results show that those two lesions were very well vascularized and their picture was similar to angioma. On the day of surgery done in local anaesthesia, the lesion on the back was removed with excessive pigmented skin. The histopathological result demonstrated melanoma Breslow > $2 \mathrm{~mm}$, Clark V diagnosed. The patient was referred to the Department of Oncologic Surgery in order to perform sentinel lymph node biopsy and excision of the second tumour. Histopathological examination did not show any melanoma metastasis in the sentinel node and no melanoma at the site of the primary tumour. The histopathology of the second excised tumour revealed metastatic melanoma (Figure 2). The genetic tests did not show a BRAF mutation. No metabolically active tumours were revealed in the positron emission tomography-computed tomography (PET-CT) study. In December 2017, the patient was admitted again to the Plastic Surgery Clinic to have another skin tumour excised from the back. Histopathological examination showed another primary cutaneous melanoma, Breslow $>2 \mathrm{~mm}$, Clark V. The patient also underwent a procedure to widen the tumour

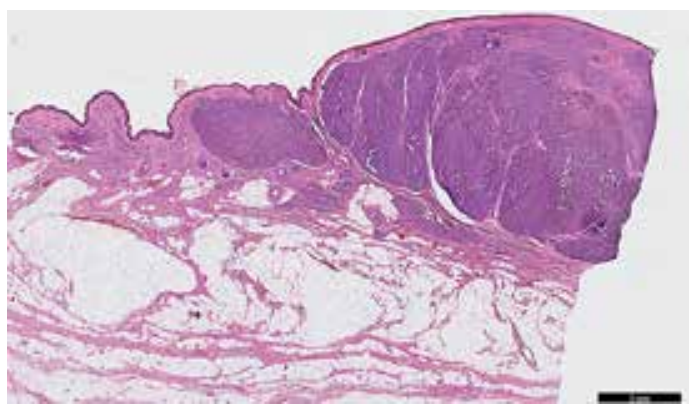

Figure 2. Histopathological view of metastasis to the skin $(\mathrm{H}+\mathrm{E})$

Address for correspondence: Marta Fijałkowska MD, PhD, Department of Plastic, Reconstructive and Aesthetic Surgery, University Hospital No. 1, 22 Kopcińskiego St, 90-153 Lodz, Poland, phone: +48 4267767 42, e-mail: fijalkowska.m@wp.pl Received: 30.10.2019, accepted: 28.01.2020. 
excision with a $2 \mathrm{~cm}$ margin, histopathological examination did not show melanoma at the site of this second primary tumour. The patient is scheduled for subsequent excision of smaller moles and till July 2019 her follow-up is preceded, the patient is being well.

In the literature we can find data suggesting that patients with giant melanocytic nevi who develop melanoma tend to have poorer prognosis than patients with melanoma de novo. It results from deeper Breslow or Clark depth, increased rate of metastasis and increased rate of positive lymph nodes on presentation [3]. Turkeltaub et al. paid attention to the fact that melanoma arising in patients with giant melanocytic nevi may be more difficult to discern and Coughlin et al. additionally underlined that melanoma in such cases may even appear beneath the skin after the giant nevi were removed $[3,4]$. Similar observations result from our paper. In the described patient, the Clark scale was the highest (V), metastasis was present in the skin, however the sentinel lymph node was negative. Diagnosed melanoma was present as a subcutaneous tumour, soft, painless on palpation, and it was more like neurofibroma or angioma.

The patients with giant congenital melanocytic nevi must be qualified for frequent physical and dermoscopic examinations. The suspected lesions should be removed and histopathological/immunohistochemical examination should be performed. If melanoma is detected, the treatment should be adequate to the degree of disease.

The patients must have very frequent follow-up examinations (at least every 2 months). Nevertheless, prognosis is uncertain in such patients.

\section{Conflict of interest}

The authors declare no conflict of interest.

\section{References}

1. Araújo C, Resende C, Pardal F, Brito C. Giant congenital melanocytic nevi and neurocutaneous melanosis. Case Rep Med 2015; 2015: 545603.

2. Tajima S, Koda K. A signet-ring cell melanoma arising from a medium-sized congenital melanocytic nevus in an adult: a case report and literature review. Pathol Int 2015; 65: 383-7.

3. Turkeltaub AE, Pezzi TA, Pezzi CM, Dao H Jr. Characteristics, treatment, and survival of invasive malignant melanoma (MM) in giant pigmented nevi (GPN) in adults: 976 cases from the National Cancer Data Base (NCDB). J Am Acad Dermatol 2016; 74: 1128-34.

4. Coughlin CC, Council ML, Gru AA, et al. Malignant melanoma arising at the site of a previously excised giant congenital melanocytic nevus. JAMA Dermatol 2014; 150: 100-1. 